\title{
Importance de la culture dans l'enseignement du lexique des émotions auprès d'étudiants mongols
}

Importance of culture in teaching lexicon of affects to Mongolian students

\section{Bita Pourmoezzi et Cristelle Cavalla}

\section{OpenEdition}

1 Journals

Édition électronique

URL : http://journals.openedition.org/tipa/3369

DOI : 10.4000/tipa.3369

ISSN : 2264-7082

Éditeur

Laboratoire Parole et Langage

Référence électronique

Bita Pourmoezzi et Cristelle Cavalla, «Importance de la culture dans l'enseignement du lexique des émotions auprès d'étudiants mongols ", TIPA. Travaux interdisciplinaires sur la parole et le langage [En ligne], 35 | 2019, mis en ligne le 23 juillet 2019, consulté le 25 juillet 2019. URL : http:// journals.openedition.org/tipa/3369; DOI : 10.4000/tipa.3369

Ce document a été généré automatiquement le 25 juillet 2019.

\section{(†) $\Theta$}

La revue TIPA. Travaux interdisciplinaires sur la parole et le langage est mise à disposition selon les termes de la licence Creative Commons Attribution - Pas d'Utilisation Commerciale - Pas de Modification 4.0 International. 


\title{
Importance de la culture dans l'enseignement du lexique des émotions auprès d'étudiants mongols
}

\author{
Importance of culture in teaching lexicon of affects to Mongolian students
}

Bita Pourmoezzi et Cristelle Cavalla

\section{Introduction}

1 Des travaux récents en sémantique, comme dans d'autres disciplines, soulignent les spécificités du lexique des émotions et des sentiments (cf. Grossmann \& Tutin, 2005). Les travaux de Clore et al. (1987) montrent ainsi, pour l'anglais, la difficulté qu'il y a à déterminer si un mot fait effectivement référence à une émotion. Il s'agit d'un champ lexical vaste et aux frontières floues: si l'on peut y inclure sans trop de discussion les expressions métaphoriques qui décrivent des affects sans utiliser spécifiquement les termes désignant les émotions (e.g. "sueurs froides »), que faut-il faire des expressions qui mettent en place une ambiance (e.g. « château hanté ») ?

2 Cependant, la fréquence de ce champ lexical le rend important dans le domaine de la didactique des langues étrangères. Il s'agit donc ici de mener une réflexion sur l'enseignement de ce lexique à des apprenants de français langue étrangère (désormais FLE) et de proposer des pistes pour pallier les difficultés particulières que cet enseignement soulève. En effet, les travaux de Wierzbicka (e.g. 1986, 1993) ont largement montré que les concepts auxquels réfèrent les différents mots désignant une émotion ou un sentiment dans une langue donnée n'ont pas forcément d'équivalent exact dans une autre langue, et qu'au contraire une très large variabilité dans les répertoires de lexique émotionnel pouvait être observée. Cette influence de la culture sur les émotions est soulignée par ailleurs, dans un tout autre domaine, par Scherer \& Walbott (1994) et Scherer \& Brosch (2009), qui montrent que la culture induit chez les individus des biais 
les rendant plus ou moins exposés à ressentir et/ou à exprimer certaines émotions. Certaines manifestations affectives seront ainsi plus fréquemment ou plus ouvertement observées dans une culture particulière que dans une autre (Wierzbicka, 1993).

3 Ainsi, la description des manifestations émotionnelles chez un personnage est l'objet de quantité d'expressions imagées qui sont des clichés construits par les langues (Iordanskaja, 1986) et qui diffèrent d'une langue à l'autre. Ces clichés peuvent décrire tant les manifestations physiologiques résultant d'une émotion (rougeur du visage, palpitations) que les manifestations socialement conditionnées pour exprimer une émotion donnée (lever les bras au ciel) (ibid.). Dans l'esprit des travaux de Mel'čuk, de telles expressions ont été décrites comme autant de fonctions lexicales permettant d'exprimer les états émotionnels des personnages. On citera ainsi les travaux de Iordanskaja (1986), Iordanskaja \& Mel'čuk (1997), Goossens (2005), Tutin et al. (2006). Ces travaux permettent, au-delà du recueil et du classement de ces expressions, d'analyser dans le détail leurs variations sémantiques et constituent un outil puissant pour l'enseignement.

4 Notre problématique se situe donc dans la lignée de ces travaux : il s'agit d'enseigner le vocabulaire (au sens large) des sentiments (Cavalla, 2006) à un public d'apprenants de FLE en Mongolie en 2009. Notre défi ici consiste à mener cet enseignement à l'étranger avec des étudiants qui ne sont pas au contact de la culture française hors des cours, ce contact restant évidemment le privilège d'un enseignement en immersion.

5 Les hypothèses qui guident ce travail sont de deux ordres : (1) l'enseignement de ce lexique pourrait aider à mieux rédiger des textes de fiction dans lesquels les personnages éprouvent des sentiments, (2) Pour exprimer la variété, la subtilité et l'intensité des émotions ressenties, il faut avoir accès à un vocabulaire imagé basé sur des formules semi figées, des collocations ou des constructions métaphoriques et qui permettent l'installation d'une ambiance (voir Boch \& Cavalla, 2005; Berdal-Masuy, 2015 ; Cavalla, 2015). Les objectifs pédagogiques des séquences visent donc: (1) l'acquisition des expressions de sentiments; (2) la distinction de différents éléments d'ambiance des expressions de sentiments (" fantôme », " avoir la chair de poule ») ; (3) l'amélioration de la mise en texte des sentiments.

6 Les expressions de sentiments qui seront abordées dans ce travail sont de trois types :

1. les lexies des sentiments : «inquiétude, angoisse »

2. les collocations : « être submergée de joie.» (Tutin et al., 2006)

3. les expressions figées : « il a la gorge nouée » (Cavalla et al. 2005)

7 L'analyse proposée par Grossmann et al. (2005) de ces trois catégories d'expressions au travers de corpus littéraires sert de support dans le présent travail en vue de la création de scenarios pour l'enseignement de ce lexique.

8 Nous nous attacherons à décrire la méthodologie mise en place pour l'enseignement de ces trois types d'éléments et à en proposer une évaluation au regard des spécificités sémantiques associées aux expressions étudiées. Il n'est pas question ici de réaliser un enseignement exhaustif de toutes ces expressions - nous nous concentrerons donc sur les expressions de la peur et de la tristesse. 


\section{Expérimentation pédagogique}

9 Cet enseignement du lexique des sentiments a été mené auprès d'étudiants adultes mongols. Deux classes ont été choisies pour cette expérimentation: un groupe de 10 étudiants de niveau B1 pour la séquence sur la tristesse et un groupe de 10 étudiants de niveau A2 pour la séquence sur la peur (niveaux du CECRL). Les cours avaient lieu dans un contexte linguistique et culturel homogène, les étudiants ayant tous la langue mongole en L1.

\section{Contenus \& supports d'enseignement}

Les séquences sont principalement basées sur l'ouvrage d'enseignement du vocabulaire des émotions-sentiments de Cavalla \& Crozier (2005). Le but de ces cours était, comme celui de cet ouvrage, d'améliorer chez les apprenants l'usage du vocabulaire des émotions - sentiments pour la description des émotions des personnages, lors de tâches écrites. La liste du vocabulaire spécifique introduit par la méthode est résumée dans le tableau 1. En plus de l'utilisation de cet ouvrage, des documents supplémentaires ont été utilisés. Il s'agissait de trois courts métrages d'animation extraits d'un DVD du CAVILAM : « une histoire vertébrale » (Clapin, 1993), «Signe de vie » (Demuynck, 2004) et « A quoi ça sert l'amour» (Clichy, 2003); quelques activités supplémentaires à celles proposées par l'ouvrage de référence ont aussi été réalisées (cf. infra).

11 L'ensemble du vocabulaire introduit par l'ouvrage de référence pour chacun des deux thèmes considérés a été présenté aux apprenants pendant la séquence, sans effectuer de sélection a priori. Cela a permis d'une part une observation fine des difficultés spécifiques soulevées par les différentes catégories de lexique, et d'autre part de fournir aux apprenants un lexique varié et détaillé, afin qu'ils aient la possibilité de le sélectionner au mieux de leurs compétences lors de la production finale.

\section{Séquence d'enseignement consacrée à la tristesse}

12 Nous avons introduit un ensemble de vocabulaire sur l'amour et la tristesse à partir d'un support audiovisuel, afin de mettre les apprenants dans l'ambiance du sentiment. Dans cette optique, un document vidéo a été présenté aux apprenants en début de séquence (Clichy, 2003). Ce document a permis une première sensibilisation des apprenants à ce sentiment, ainsi qu'un rappel de leur connaissance préalable du lexique qui lui est lié. Cette démarche est innovante dès l'instant où l'enseignant tient compte de l'aspect psychologique de l'introduction de ce type de lexique dans une classe de langue. Ceci pour inciter les apprenants à produire un discours en faisant appel à l'ambiance préalablement créée et aux ressentis propres à leur culture. Les apprenants étaient donc en confiance pour la tâche d'évaluation initiale. La séquence pédagogique débute par la rédaction d'un texte à partir d'un court-métrage sans parole et se termine par une production finale sur un autre court-métrage abordant le même sentiment. La production initiale permet d'évaluer la connaissance lexicale et le savoir de mise en texte des apprenants.

13 La suite de la séquence sur la tristesse s'est déroulée selon la progression proposée par l'ouvrage de Cavalla \& Crozier (2005). Le temps consacré à ce thème correspond à 8 
heures de cours, plus les 2 heures de production écrite finale. Toutes les activités de l'ouvrage ont été réalisées en cours (sauf l'activité 118 liée au concept de joie). Des activités supplémentaires présentant les variations d'intensité et de durée de la tristesse dans ces expressions ont été proposées car les apprenants avaient du mal à maîtriser ces subtilités. Par exemple, les apprenants sont invités à réutiliser les expressions à l'oral, à classer les expressions de chaque sentiment selon leur degré d'intensité puis à trouver une situation correspondant à l'expression choisie. Une comparaison des expressions françaises avec celles de leur langue a permis un travail sur les représentations culturelles de ces sentiments et d'en observer et les similitudes et les différences. Ces exercices permettent de mieux comprendre les nuances d'intensité du sentiment en contexte.

Pour la production finale, un court métrage muet (Demuynck, 2004) mettant en scène une femme brisée par la perte de son mari a été projeté aux apprenants, qui devaient ensuite en décrire l'histoire. La tâche de cette production finale est similaire à la tâche initiale : il s'agit dans les deux cas de décrire une histoire qu'ils viennent de visionner, histoire évoquant clairement des sentiments tristes.

Tableau 1. Comparaison des entrées lexicales entre un ouvrage et les productions des apprenant

\begin{tabular}{|l|l|l|l|}
\hline & & Peur & Tristesse \\
\hline \multirow{4}{*}{ Ouvrage Cavalla \& Crozier } & 29 & 29 \\
\hline \multirow{4}{*}{ Vocabulaire } & nb. d'entrées ES & 30 & 29 \\
\cline { 2 - 5 } & nb. moyen / ap. & 5,8 & 6,7 \\
\hline & min / max & $2 / 10$ & $1 / 10$ \\
\hline \multirow{2}{*}{ Eléments d'ambiance } & nb. moyen / ap. & 5 & 5,6 \\
\cline { 2 - 5 } & nb. d'entrées ES & 33 & 25 \\
\cline { 2 - 5 } & min / max & $1 / 13$ & $2 / 8$ \\
\hline
\end{tabular}

Le tableau 1 présente une comparaison entre le nombre d'entrées des noms d'émotions dans l'ouvrage Emotions-Sentiments (Cavalla et Crozier, 2005) et le nombre d'entrées moyenne par apprenants de ces mêmes noms. Les lignes «min / max » indiquent le nombre minimum et maximum d'entrée pour un apprenant. Il s'agit du vocabulaire des émotions ainsi que des éléments d'ambiance, respectivement pour la peur et pour la tristesse.

\section{Séquence d'enseignement consacrée à la peur}

La séquence sur la peur a suivi intégralement la progression proposée par l'ouvrage de Cavalla \& Crozier (2005). L'ambiance est installée par la diffusion d'un enregistrement sonore comportant des bruits de vent, des cris, etc., tiré du CD de l'ouvrage. Ces sons ont unanimement évoqué le sentiment de peur chez les apprenants. Les apprenants ont été 
dispensés de la production écrite initiale en raison de leur niveau (A2). La séquence a duré 8 heures, plus les deux heures consacrées à la production écrite finale.

Le vocabulaire de la peur, et en particulier les expressions d'angoisse et d'inquiétude, présente des variations sémantiques subtiles. Leur enseignement est donc délicat et leur utilisation pertinente par les apprenants aussi. Durant cette séquence, les apprenants ont donc montré une plus grande aisance pour manier des métaphores basées sur des manifestations physiologiques (trembler de peur, etc.) que des lexies sémantiquement complexes (terreur, angoisse, frayeur). Dans certains cas simples, des activités de mémorisation du vocabulaire (restitution orale d'expressions apprises en cours lors de tours de table ; séances de mime d'expressions que l'assistance doit deviner, etc.) ont été réalisées, afin d'aider les étudiants à mieux le fixer.

Figure 1. Vocabulaire de la peur (à gauche) et de la tristesse (à droite) utilisé dans les productions finales des apprenants

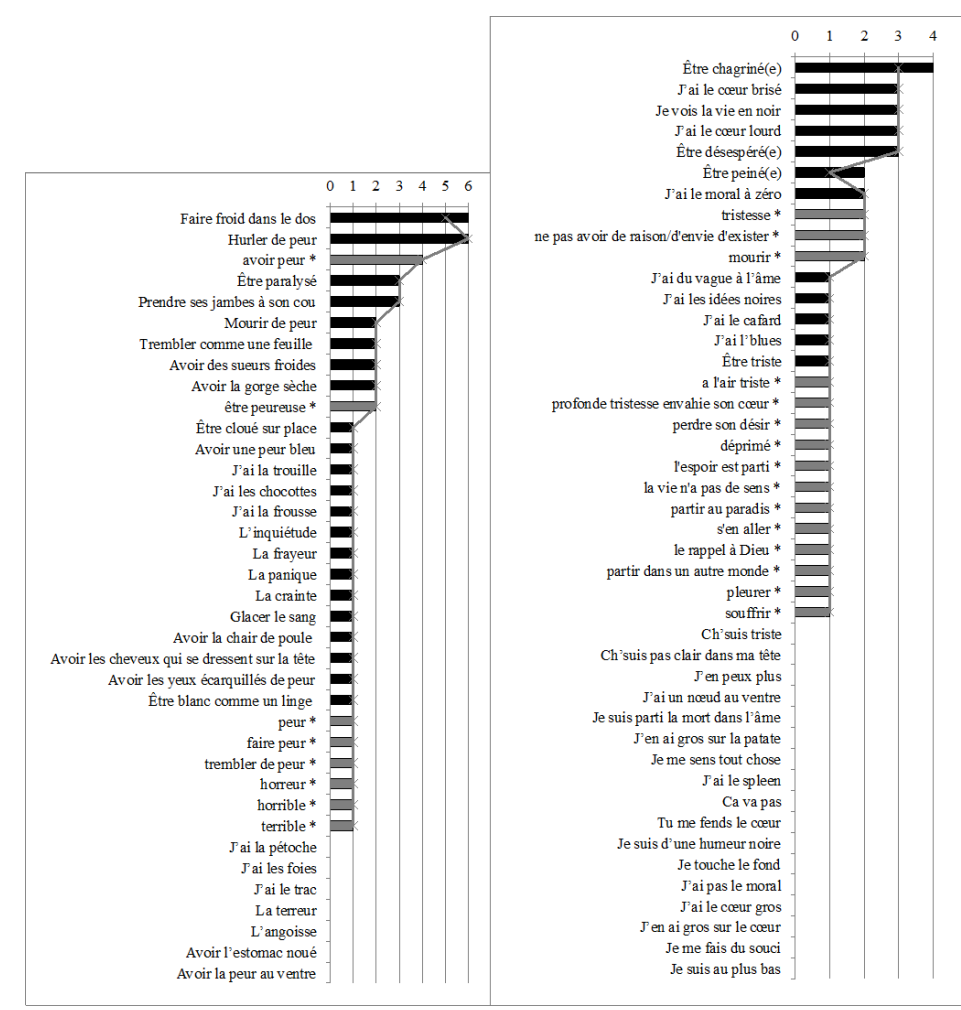

Dans la figure 1, les entrées sont classées par ordre décroissant d'utilisation par les apprenants. Les entrées supplémentaires introduites par les apprenants sont repérées par un astérisque et correspondent aux barres grises. Les entrées associées à une fréquence nulle ont été vues en cours mais ne sont pas utilisées dans les productions. Les barres indiquent le nombre total d'utilisations de chaque entrée lexicale, tandis que le trait grisé indique le nombre d'apprenants différents qui ont utilisé une entrée donnée. 
Figure 2. Expressions d'ambiance utilisées lors des productions finales

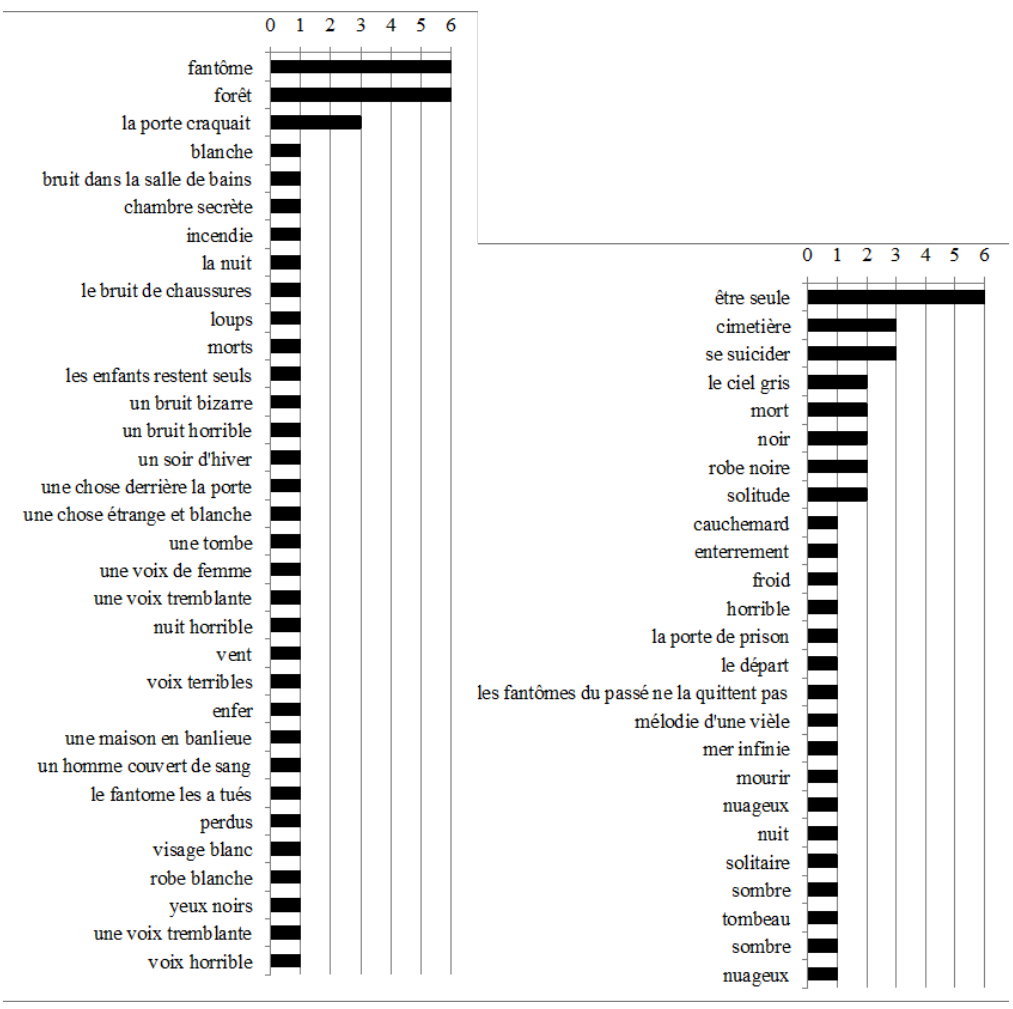

19 Cette figure 2 contient le vocabulaire de la séquence sur la peur (à gauche) et sur la tristesse (à droite) dans les productions finales des apprenants. Les barres indiquent le nombre d'utilisation de chaque entrée.

La production finale est basée sur l'exercice de clôture de la séquence pédagogique proposé par l'ouvrage de Cavalla \& Crozier (2005). Il s'agit d'écrire une histoire « qui fait peur ", en décrivant le cadre et le ressenti du personnage, grâce au lexique vu en cours.

\section{Analyse des productions des apprenants}

21 Une analyse quantitative des productions des apprenants à la fin des séquences pédagogiques permet de valider leurs acquis (cf. Boch \& Cavalla, 2005). Cette analyse est résumée par les schémas des figures 1 et 2 et par le tableau 1 qui en fait la synthèse.

Ces comptages montrent une meilleure maitrise du vocabulaire enseigné dans le cas de la peur : la plupart (76\%) des entrées vues en cours ont été réutilisées et les éléments d'ambiance sont nombreux et variés. Par contre dans le cas de la tristesse, plus de la moitié (59\%) des entrées vues en cours n'ont pas été utilisées. D’autres ont été employées, mais montrent moins de précision et de variabilité. Par ailleurs, les éléments d'ambiance sont eux aussi moins nombreux (33 contre 25).

Une analyse qualitative confirme ces résultats: on observe des difficultés plus importantes à produire des textes exprimant de manière efficace le sentiment étudié quand il s'agit de la tristesse, comparée à la peur. Les textes de la séquence "tristesse » sont clairement moins évocateurs que ceux de la séquence " peur ». Même s'ils emploient une quantité globalement comparable de vocabulaire dédié, le nombre moins important d'éléments d'ambiance, ainsi que l'emploi moins pertinent des expressions, donne une 
impression globale clairement différente pour les deux ensembles de productions. Cette différence n'est pas expliquée par la différence de niveau des deux groupes, puisque c'est le groupe le plus avancé qui a travaillé sur la tristesse, et la durée des deux séquences d'enseignement était la même.

Cette comparaison entre les productions de peur et de tristesse ne doit pas donner un éclairage trop négatif sur les résultats obtenus avec la tristesse. Une analyse des textes de la production initiale, réalisés lors de la séquence sur la tristesse permet de constater les difficultés importantes des apprenants pour exprimer ce sentiment à l'écrit. Ainsi, on constate une absence presque complète de mise en texte: les productions initiales sont constituées de phrases isolées qui, au-delà des problèmes de français, permettent difficilement de suivre la progression de la narration. En terme de vocabulaire, seules deux occurrences de lexique dédié ont pu être relevées ("il est triste») et un seul élément d'ambiance ( il est seul »). On relève cependant des tentatives d'exprimer les émotions, mais elles butent sur un manque de vocabulaire approprié : on observe ainsi plusieurs métaphores qui sont vraisemblablement des traductions directes du mongol en français (par exemple «il poussait un cri au monde de nouveau»), mais qui sont tout à fait inappropriées pour exprimer le sentiment recherché en français. L'emploi de tels tropes révèle le désir de créativité qui faute de connaissance linguistique ne peuvent être acceptés en l'état en français. Par ailleurs, la comparaison des productions finales montre un accroissement qualitatif net. On obtient des textes de meilleure qualité qui, s'ils manquent encore de maîtrise, parviennent à exprimer les idées visées avec un vocabulaire français. Il n'y a plus de recherche de solution en langue maternelle et la plupart des textes sont à peu près structurés même si les expressions figées ne sont pas toujours employées à bon escient. L'exemple suivant : «j'ai le cœur brisé, je veux mourir car j'ai le cafard. » montre un évident manque de logique entre le ressenti et les réactions du personnage.

Cette comparaison entre productions initiales et finales ne peut pas être effectuée pour la séquence de la peur, car les premières n'ont pas été réalisées par les apprenants. Cependant, la maîtrise de la plupart du vocabulaire introduit durant les cours et l'utilisation d'un nombre important d'éléments d'ambiance adéquats à l'émotion traitée montre une bonne acquisition des concepts culturels comme du vocabulaire spécifique. La qualité des productions finales plaide aussi dans ce sens.

\section{Discussion \& Conclusion}

La comparaison des analyses qualitative et quantitative permet d'arriver à la conclusion suivante : l'utilisation intensive du vocabulaire appris durant la séquence ne suffit pas à écrire un texte qui rende clairement l'impression émotionnelle recherchée. Cela rejoint les conclusions de Cavalla (2015) et Berdal-Masuy (2015). En effet, les productions de tristesse comme de peur utilisent globalement une quantité comparable de lexique dédié, mais les secondes sont qualitativement plus efficaces à transmettre le ressenti de peur, et ceci ne peut être expliqué par le niveau des apprenants.

L'utilisation dans un texte du vocabulaire appartenant au champ lexical d'un sentiment ne suffit pas à l'évoquer. Encore faut-il l'employer dans un contexte adéquat à ses caractéristiques sémantiques. L'utilisation d'éléments d'ambiance aide à atteindre ce but (ils sont plus nombreux dans le cas des textes traitant de la peur). De même, le meilleur texte sur la tristesse produit par les apprenants emploie moins d'expressions désignant 
précisément ce sentiment que certains autres textes, mais l'idée de tristesse y est contextualisée, l'ambiance est rendue par un ensemble de périphrases, par des descriptions complexes qui font référence à l'idée de tristesse, même sans référence directe. Le résultat est clairement supérieur à d'autres productions qui s'apparentent plus à un catalogue d'expressions, rendues inefficaces car utilisées de manière inadéquate dans la structure de récit.

Parce que le sens précis de ces expressions leur reste encore inaccessible, les apprenants ne sont pas à l'aise pour les utiliser dans le cas de la tristesse, tandis que pour la peur, les expressions sont bien comprises et donc utilisées à bon escient. Cela influence directement la qualité textuelle de leurs productions finales.

Pourquoi des apprenants de niveau supérieur auraient plus de mal à assimiler le sens précis du vocabulaire spécifique d'un sentiment (la tristesse) qu'un autre groupe pour un second sentiment (la peur) ? Il nous semble que la réponse à cette question peut venir de la nature des expressions utilisées pour ces deux sentiments. Si l'on se réfère à la figure 1 , on peut constater une différence claire entre les deux listes: le lexique de la peur fait majoritairement appel à des expressions décrivant les effets physiologiques de la peur (sueurs froides, trembler comme une feuille, estomac noué, gorge sèche...), tandis que le lexique de la tristesse est constitué presqu'exclusivement des expressions faisant directement référence à des nuances de tristesse (j'ai le cœur brisé, j'ai l'blues...), sans presque jamais utiliser d'expressions impliquant le corps (sauf «j'ai un nœud au ventre » qui de plus renvoie à «l'estomac noué » de la peur). Cette différence est aussi illustrée dans la méthode par l'absence d'image représentant l'état émotionnel de la tristesse, quand les illustrations sont fréquentes pour la séquence sur la peur. Cette différence entre peur et tristesse peut aussi se retrouver dans le modèle de Scherer (1986), qui postule une différence dans les réponses physiologiques en fonction de la durée des phénomènes affectifs - et la tristesse est un sentiment plus à même de durer que la peur (sur l'importance du corps dans le ressenti émotionnel, voir aussi Damasio, 1998).

Cette différence de nature des expressions utilisées pour ces deux sentiments semble donc importante pour expliquer nos résultats. Ainsi, comme le montrent très bien les travaux de Iordanskaja (1986) pour le russe, les expressions métaphoriques décrivant les symptômes des émotions peuvent varier largement d'une culture à l'autre, d'autant plus lorsque les expressions réfèrent à des manifestations sociales plutôt qu'à des manifestations physiologiques. Cependant, cette variation informe surtout sur les différences culturelles qui peuvent exister entre deux langues (cf. Wierzbicka, 1993, pour une comparaison entre russe et anglais). Pour autant, et malgré les différences d'évaluation émotionnelle liées aux cultures (Scherer \& Brosch, 2009), les manifestations physiologiques de ces émotions seraient universelles (ibid; Scherer, 1986). Il pourrait ainsi être plus aisé à un apprenant d'appréhender le sens d'une expression métaphorique dénotant le symptôme physique d'une émotion qu'il aura déjà pu expérimenter lui-même (et/ou pour laquelle il aura pu voir une image parlante), plutôt que de se souvenir d'un signe abstrait. Ainsi, durant des exercices de microproductions réalisés pendant les deux séquences, des étudiants ont spontanément proposé des expressions libres destinées à exprimer le sentiment étudié, expressions traduites directement du mongol : il s'agissait d'expressions imagées qui ne fonctionnaient pas en français, mais dont la motivation était claire. De ce point de vue, le lexique de la tristesse, doté de peu de références à des manifestations physiques de l'émotion, pourrait être plus complexe à intégrer et donc 
être à l'origine d'une partie des difficultés rencontrées par les apprenants pour l'utiliser dans un contexte adéquat.

En conclusion de ce travail, nous voudrions souligner l'importance de la mise en texte pour développer à l'écrit l'expression des émotions et des sentiments : les termes doivent être employés dans des contextes adéquats pour être interprétés justement. Ainsi, si la maîtrise d'une quantité minimale de vocabulaire est requise, il ne semble pas nécessaire d'en maitriser une importante quantité, mais plutôt de bien apprendre où et comment l'utiliser : savoir trouver «le bon endroit au bon moment »- et cela est surtout vrai à l'écrit.

Par ailleurs, les similitudes et les différences culturelles peuvent être une piste intéressante à étudier pour mettre en place un enseignement du lexique des émotionssentiments qui permette aux apprenants de bien saisir les variations subtiles de sens. Pour cela, les cadres émotionnels proposés par Cavalla (2015), peuvent se révéler un outil performant pour présenter l'atmosphère liée au lexique de l'émotion enseignée. Une fois certains concepts sémantiques bien assimilés par les apprenants, il sera ainsi possible de se concentrer sur la mise en texte afin de mieux leur faire travailler la cohérence textuelle de leurs productions.

Un enseignement efficace du lexique des émotions-sentiments devra ainsi proposer un programme pédagogique spécifique pour chaque type de sentiment. Il devra être à même d'insister plus ou moins sur les différents aspects du vocabulaire : il sera donc important de produire des séquences adaptées à chaque affect, en considérant l'aspect culturel de ce lexique spécifique, les ambiances qui lui sont culturellement liées, la fréquence d'emploi de telle ou telle expression par un locuteur natif, les éventuelles descriptions de manifestations physiques et le caractère plus ou moins universel des concepts utilisés, en fonction des langues et des cultures des apprenants.

\section{BIBLIOGRAPHIE}

Berdal-Masuy, F. (2015) Enseigner l'expression orale des émotions en classe de FLE : quel lexique et quel dispositif ?, Le Langage et l'Homme, 50-2, p. 129-145.

Boch, F. \& C. Cavalla (2005) Evaluer l'expression des sentiments dans des textes d'enfants, une mission impossible ?, Repères, 31, p. 55-71.

Cavalla, C. (2006) Lexique et représentation des sentiments, in Louis, V., Auger, N. \& I. Belu (eds) Former les professeurs de langues à l'interculturel - À la rencontre des publics, Louvain-la-Neuve, CortilWodon: E.M.E.

Cavalla, C. (2015) Quel lexique pour quelles émotions en classe de FLE ?, Le Langage et l'Homme, 50-2, p. 115-128.

Cavalla, C. \& E. Crozier (2005) Emotions - Sentiments : nouvelle approche lexicale du FLE, Grenoble: PUG, $104 \mathrm{p}$.

CECRL (2001) Cadre Européen Commun de Référence pour les Langues, Paris: Didier. 
Clapin, J. (1993) Une histoire vertébrale, Court métrage d'animation, Sortie le 06 Octobre 1993, durée : 9 min.

Clichy, L. (2003) À quoi ça sert l'amour ?, Court métrage d'animation, Sortie en 2003, Studios Cube Creative Computer, durée : 3 min.

Clore, G.L., Ortony, A. \& M.A. Foss (1987) The psychological foundations of the affective lexicon, Journal of Personality and Social Psychology, 53(4), p. 751-766.

Damasio, A.R. (1998) Emotion in the perspective of an integrated nervous system, Brain Research Review, 26, p. 83-86.

Demuynck, A. (2004) Signe de vie, Court métrage d'animation, Sortie en 2004, durée : $8 \mathrm{~min}$.

Iordanskaja, L. (1986) Russian expressions denoting physical symptoms of emotions, Lingua, 69, p. 245-282.

Iordanskaja, L. \& I. Mel'čuk (1997) Le corps humain en russe et en français, Vers un Dictionnaire explicatif et combinatoire bilingue, Cahiers de lexicologie, 70(1), p. 103-135.

Goossens, V. (2005) Esquisse de typologie sémantique fondée sur les collocations verbales, Lidil, 32, p. 103-121.

Grossmann, F. \& A. Tutin (2005) Sémantique des noms et adjectifs d'émotion, Lidil, 32, p. 5-15.

Scherer, K. R. (1986) Voice, stress, and emotion, in Appley, M.H. \& R. Trumbull (eds) Dynamics of stress, New York: Plenum Press, p. 157-179.

Scherer, K.R. \& T. Brosch (2009) Culture-specific appraisal biases contribute to emotion disposition, European Journal of Personality, 23, p. 265-288.

Scherer, K.R. \& H.G. Walbott (1994) Evidence for universality and cultural variation of differential emotion response patterning, Journal of Personality and Social Psychology, 66(2), p. 310-328.

Tutin, A., Novakova, I., Grossmann, F., \& C. Cavalla (2006) Esquisse de typologie des noms d'affect à partir de leurs propriétés combinatoires, Langue française, 150, p. 32-49.

Wierzbicka, A. (1986) Human emotions: universal or culture-specific?, American Anthropologist, new series, 88(3), p. 582-594.

Wierzbicka, A. (1993) A conceptual basis for cultural psychology, Ethos, 21(2), p. 205-231.

\section{RÉSUMÉS}

Cet article relate deux expérimentations autour de l'enseignement du lexique des émotions en classe de FLE auprès d'étudiants mongols en Mongolie de niveau A2 et B1 (CECRL, 2001). Ces expérimentations ont de créatif l'introduction d'une atmosphère autour des émotions évoquées : la peur et la tristesse. Nous montrerons comment les étudiants articulent lexique appris et description d'une atmosphère permettant de déceler l'émotion attendue. En outre, les textes produits révèlent une certaine créativité de la part des apprenants, mais aussi des aspects culturels non négligeables pour l'expression des émotions. Nous tenterons ici d'expliquer ces phénomènes à l'aune des résultats d'analyse des textes tant par des aspects quantitatifs (statistiques fréquence d'utilisation des mots) que par des aspects qualitatifs (analyse linguistique des textes). En conclusion, nous aborderons les aspects culturels inhérents à ce lexique spécifique des émotions en comparant notamment les manifestations universelles physiologiques et les influences culturelles relatives à chacune des émotions choisies. 
This article describes two experiments on the teaching of the lexicon of affects in FFL (French as a Foreign Language) class with Mongolian students of A2 and B1 accordinge to the Common European Framework of Reference for Languages (CECRL, 2001) in Mongolia. Recent works in semantics, as in other disciplines, underlines the specificities of the lexicon of affects and feelings (cf. Grossmann \& Tutin, 2005). The frequency of use of this lexicon makes it important in the field of foreign language didactics.

We will be reflecting the teaching of this lexicon to learners of French as a foreign language and to suggest ways to overcome the particular difficulties that such a teaching method raises.

The underlying hypothesis of this work is twofold: (1) the teaching of this lexicon should enable learners to better write fictional texts in which the characters feel emotions (2) To express the variety, the subtlety and the intensity of the emotions felt, it is necessary to have a imaged vocabulary and expressions based on collocations, idioms or metaphorical constructions wich allow to set up an atmosphere related to the taught emotion (see Boch \& Cavalla, 2005 ; BerdalMasuy, 2015 ; Cavalla, 2015). Of course, it should be noted that we are not discussing of an exhaustive teaching of all these expressions.

The educational objectives of the sequences are (1) the acquisition of expressions of emotions; (2) the distinction of different mood elements from expressions of emotions ("fantôme " [ghost], « avoir la chair de poule» [having goose bumps], for example); (3) improving the setting in text of emotions. The purpose of these courses is therefore to improve the learners' use of the vocabulary of emotions - affects for the description of the emotions of the characters, during written tasks.

Having this in mind, we introduced a set of vocabulary explaining fear and sadness from audio and audiovisual media, to put learners in the mood of those affects. These documents made it possible for the learners to be first aware of this emotion, as well as a reminder of their previous knowledge of the lexicon related to it. This approach is innovative as soon as the teacher takes into account the psychological aspect of the introduction of this type of lexicon into a language class. This is to encourage the learners to produce a speech by appealing to the atmosphere previously created and the feelings of their own culture.

The expressions of feelings discussed in this work are of three types:

(1) The lexicon of affects: « inquiétude, angoisse » [anxiety, distress]

(2) The collocations: « être submergée de joie » [to be overwhelmed with joy] (Tutin et al., 2006)

(3) The idioms: « il a la gorge nouée » [he felt a lump in his throat](Cavalla et al., 2005)

The analysis proposed by Grossmann et al. (2006) of these three categories of expressions through literary corpus serves as a support in the present work for the creation of scenarios for the teaching of this lexicon.

These experiments have creative introduction of an atmosphere around the evoked emotions: fear and sadness, we will show how students articulate learned lexicon and description of an atmosphere to detect the taught emotion. In addition, the texts produced reveal a certain creativity from the learners' side, but also significant cultural aspects for the expression of emotions. We will attempt here to explain these phenomena in terms of the results of text analysis both quantitative aspects (statistics of use of words) and qualitative aspects (linguistic analysis of texts). A quantitative analysis of the learners' productions at the end of the pedagogical sequences validates their achievements (see Boch \& Cavalla, 2005). This analysis is summarized by the diagrams of Figures 1 and 2 and by Table 1 , which synthesizes them. A qualitative analysis makes it possible to observe ease and difficulties in writing texts that effectively express the emotions studied by comparing them.

This comparison allows us to address the cultural aspects inherent to this specific the lexicon of affects by comparing in particular the universal physiological manifestations and the cultural influences relating to each of the chosen emotions. We observe several metaphors that are likely 
to be direct translations from Mongolian into French (for example "he shouted to the world again" to express the sadness), but which are quite inappropriate to express the emotion sought in French. The use of such tropes reveals the desire for creativity that lack of linguistic knowledge cannot be accepted in French.

As Iordanskaja's (1986) work for Russian clearly shows, the metaphorical expressions describing the symptoms of emotions can vary widely from one culture to another, especially when expressions refer to social manifestations rather than to physiological manifestations.

However, this variation mainly informs the cultural differences that can exist between two languages (see Wierzbicka, 1993, for a comparison between Russian and English). Considering the above, and despite cultural differences in emotional evaluation (Scherer \& Brosch, 2009), the physiological manifestations of these emotions are universal (Scherer, 1986). It could therefore be easier for a learner to grasp the meaning of a metaphorical expression denoting the physical symptom of an emotion that he has already been able to experience himself and/or for which he could have seen a talking image (cold sweat, shaking like a leaf), rather than remembering an abstract sign (to feel blue) almost never use expressions involving the body. In a result, during micro-productions exercises performed during the two sequences, students spontaneously proposed free expressions intended to express the taught emotion, expressions translated directly from Mongol: they were imaged expressions that did not work in French, but whose motivation was clear. From this point of view, the lexicon of sadness, with few references to physical manifestations of emotion, could be more complex to integrate and therefore be at the root of some of the difficulties encountered by learners to use it in a proper context.

In conclusion of this work, we will emphasize the importance of the setting in text to develop the expression of affects in the writing: the terms must be used in contexts that are appropriate to be interpreted correctly. Therefore, if the mastery of a minimum number of vocabulary is required, it does not seem necessary to control a large number, but rather to learnthe lexical associations of the affects studied and where and how to use it: find "the right place at the right time" - and this is especially true in writing.

On the other hand, similarities and cultural differences can be an interesting subject to study in order to set up a lexicon of affects -feelings that allows learners to grasp the subtle variations of meaning. For that, the emotional frameworks proposed by Cavalla (2015), can be a powerful tool to present the atmosphere related to the lexicon of the taught emotion. Once some semantic concepts are well assimilated by the learners, it will be possible to focus on the text in order to better make them work the coherence of their writings.

An effective teaching method of the lexicon of affects-feelings will have to suggest a specific educational program for each type of affect. The method will have to be able to insist more or less on the different aspects of the vocabulary: it will be important to produce sequences adapted to each affect, considering the cultural aspect of this specific lexicon, the ambiances which are culturally related, the frequency of use of this or that expression by a native speaker, the possible descriptions of physical manifestations and the more or less universal character of the concepts used, depending on the languages and cultures of the learners.

\section{INDEX}

Mots-clés : français langue étrangère, émotion, culture, lexique, créativité

Keywords : French as a foreign language, affect, culture, lexicon, creativity 


\section{AUTEURS}

\section{BITA POURMOEZZI}

Université Sorbonne Nouvelle-Paris3, DILTEC EA2288

bita.rilliard@gmail.com

\section{CRISTELLE CAVALLA}

Université Sorbonne Nouvelle-Paris3, DILTEC EA2288

cristelle.cavalla@sorbonne-nouvelle.fr 